\title{
Comparative Analysis and Survey of LMS and RLS Adaptive Algorithms
}

\author{
Farheen Ali \\ Truba College of Science and \\ Technology, Bhopal, M.P., India
}

\author{
Paresh Rawat \\ Dept. of ECE, Truba College of \\ Science and Technology, Bhopal, \\ M.P., India
}

\author{
Sunil Malvia \\ Dept. Of ECE, Truba College of \\ Science and Technology, Bhopal, \\ M.P., India
}

\begin{abstract}
This review paper is surveyed in different concerns. It has been conducted to know about designing of adaptive filter and also to know where the adaptive algorithms are used in the different applications. The main goal of this review paper is to study and performance of different adaptive filter algorithms on the basis of literature survey.
\end{abstract}

\section{Keywords}

LMS algorithms, RLS Algorithm, Adaptive Filter, mean state error, Digital Filter, Digital signal processing.

\section{INTRODUCTION}

A literature survey clearly establishes the need of the work. It helps to address queries regarding development in these studies and allow it for many unresolved problems to happen and thus obviously state all boundaries about the growth of the research work. Digital signal processing (DSP) systems are becoming more and more motivating due to the development in digital circuit design[1]. There are some schemes which include digital systems for various filtering application. Digital systems are used to control the information of the various input signal. Adaptive filters are made appropriate in any type of new environment. Adaptive filter is used for digital signal processing and also maintain several applications in time varying environment of input statistics. Faulty signal is enhanced by certain and uncertain noise which is decreases with the help of adaptive filters. The applications of adaptive filters such as inverse modeling, identification, interference cancellation and prediction are the main essentials to solve the problem of noise and acoustic echo cancellation. There are several algorithms are designed mostly LMS and RLS algorithm for interference cancellation to get appropriate adaptive filter. The performance measures of adaptive algorithm are rate of convergence, computational requirements, numerical robustness and stability. Section 2 gives an overview of adaptive filter. The brief description of various literature surveys is described in section 3. Section 4 gives the comparison between adaptive algorithms such as Least Mean Square (LMS), and Recursive Least Square (RLS). Section 5 concludes the main research work.

\section{BACKGROUND AND MOTIVATION OF RESEARCH}

In the field of signal processing, there is a major requirement for a special class of digital filters known as adaptive filters. Adaptive filters are used generally in many different configurations, and for assured applications these filters have a great benefit over the standard digital filters. They can adapt their filter coefficients to the environment according to predetermined rules. The filters are capable of learning from the statistics of current situations, and modify their coefficients in order to achieve a certain goal. They are used in many different situations when it is not possible or inconvenient to use a predesigned filter. In order for a filter to be designed, awareness of the desired response is required a priori. When such knowledge does not exist, due to the changing nature of the filter's requirements, it is not possible to design a standard digital filter. In such situations, adaptive filters are popular. Adaptive filters continuously modify their impulse response in order to satisfy the given conditions, by doing so, alter the very characteristic of their response.

There are two module of adaptive filtering algorithms namely Recursive Least Squares (RLS)[2] and Least Mean Squared (LMS) [3] are capable of performing the adaptation of the filter coefficients. Every algorithm presents certain advantages and disadvantages with regards to their implementation. In most cases, the premium choice of the algorithm depends on trade-offs between implementing costs and performance gains. Therefore, there is a constant requirement and effort across the signal processing community for improving the current algorithms in provisions of both rising the performance and reducing the computational complexity.

\subsection{Digital Filter-[4],}

A Digital Filter is a system that perform mathematical operations, usually it consists of an analog-to-digital converter to sample the input signal, followed by a microprocessor and some peripheral devices such as memory to store data and filter coefficients. And the digital-to-analog converter used to complete the output stage. Program Instructions (software) running on the microprocessor implement the digital filter by performing the essential mathematical operations on the numbers received from the ADC. In various high performance applications, an FPGA or ASIC is used instead of a general purpose microprocessor, or a DSP with specific paralleled architecture for filtering.

\subsection{Adaptive Filter-[5],}

An adaptive filter is a computational device used for mathematical operations, that iteratively shows the relationship between the input/output signals of the adaptive filter. An adaptive filter manually adjusts the filter coefficients according to an adaptive algorithm. The following figure shows the diagram of a typical adaptive filter[6]. 


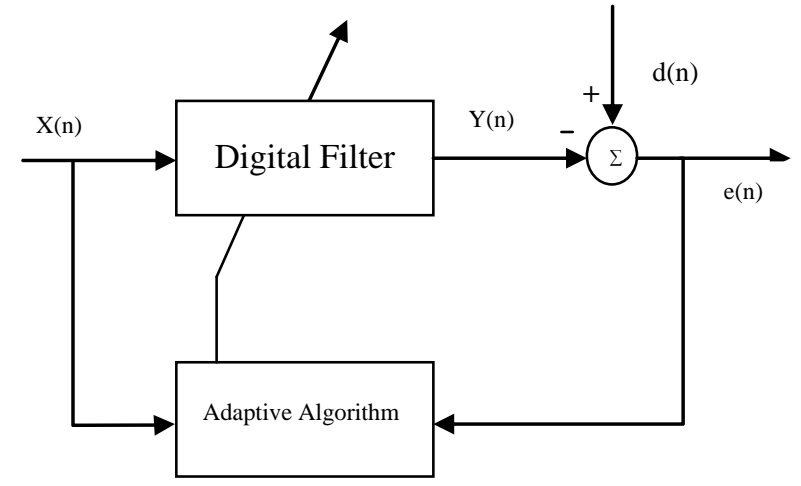

Fig. 1. Adaptive Filter [6].

Where.

$\mathrm{x}(\mathrm{n})-$ is the input signal to a linear filter at time $\mathrm{n}$.

$y(n)-$ is the output signal.

$\mathrm{d}(\mathrm{n})$ - is an input signal to the adaptive filter.

$\mathrm{e}(\mathrm{n})$ - is the error signal that denotes the difference between $\mathrm{y}(\mathrm{n})$ and $\mathrm{d}(\mathrm{n})$.

\subsection{LMS Algorithm (Least Mean Square)- [3],}

The LMS adaptive filter has a filtering section and one adaptation section. The filtering section consists of finite impulse response (FIR) filter and adaptation section consists of LMS algorithm. In every iteration, the filtering section calculates a filter output from which an error value is computed. The adaptive section uses the error value to calculate the weight-increment term for updating the weight vector for the next iteration The nth iteration weight-vector $\{w(n)\}$ of an LMS adaptive filter is updated for the $(n+1)$ th iteration using the relation:

$$
\mathrm{w}(\mathrm{n}+1)=\mathrm{w}(\mathrm{n})+\mu \cdot \mathrm{x}(\mathrm{n}) \cdot \mathrm{e}(\mathrm{n})
$$

where, $\mu$ is the convergence factor.

The N-point weight-vector $\mathrm{w}(\mathrm{n})$ is defined as

$$
\mathrm{w}(\mathrm{n})=[\mathrm{wn}(0), \mathrm{wn}(1), \ldots, \mathrm{wn}(\mathrm{N}-1)] \mathrm{T}
$$

And the error "e(n)" is calculated as

$$
\mathrm{e}(\mathrm{n})=\mathrm{d}(\mathrm{n})-\mathrm{y}(\mathrm{n})(3)
$$

where, $d(n)$ is the desired response and $y(n)$ is the an estimate of the desired response which is calculated as

$$
\mathrm{y}(\mathrm{n})=\mathrm{x}(\mathrm{n}) \operatorname{Tw}(\mathrm{n})
$$

where, the N-point input vector $\mathrm{x}(\mathrm{n})$ is defined as

$$
x(n)=[x(n) x(n-1), \ldots, x(n-N+1)] T
$$

\subsection{RLS (Recursive Least Squares)}

Algorithm- [2],[7],

The Recursive least squares (RLS) adaptive filter is an technique which recursively search the filter coefficients that reduce a weighted linear least squares cost function linking to the input signals. The RLS algorithms are known for their outstanding performance when working in time varying environments, but at the cost of an increased computational complication and some stability problems. In this algorithm the filter tap-weight vector is efficient using Equations.

$w(n)=w T(n-1)+k(n)$ en-1(n)

$$
\begin{aligned}
& \mathrm{k}(\mathrm{n})=\mathrm{u}(\mathrm{n}) /(\lambda+\mathrm{X} T(\mathrm{n}) \mathrm{u}(\mathrm{n})) \\
& \mathrm{u}(\mathrm{n})=\mathrm{w} \lambda-1(\mathrm{n}-1) \mathrm{X}(\mathrm{n})
\end{aligned}
$$

Eq. (2) and eq. (3) is intermediate gain vector used to calculate tap weights.

Where, $\lambda$ is a small positive constant very close to, but lesser than 1. The filter output is calculated using the filter tapweights of beyond iteration and the current input vector as in Equation (4).

$$
\begin{aligned}
& y n-1(n)=w T(n-1) X(n) \\
& \text { en-1(n) }=d(n)-y n-1(n)
\end{aligned}
$$

In the RLS Algorithm [1] the approximation of previous samples of output signal, filter weight and error signal is mandatory that leads to higher memory requirements.

\section{LITERATURE SURVEY}

In the earlier period, several researchers and authors have investigated the BIST testing techniques for the detection of fault coverage. In this survey paper various related literature such as IEEE transactions, other journals and proceedings of various national and international conferences were reviewed.

Pramod kumar meher et. al. [3] In this research paper researcher present an efficient architecture for the implementation of DLMS adaptive filter. While for achieving lower adaption delay, researcher used a normal practical product generator and a perfect strategy for optimized balanced pipelining across the time consuming combination block of the structure is projected. Also researcher discussed about an efficient fixed point implementation scheme of proposed architecture and drives the expression for steady state error.

S. Savitha et. al. [8] in this research paper researcher discussed about the implementation of efficient LMS adaptive filter with low adaption delay, for achieving area delay power and lower adaption delay, researcher proposed an strategy for optimized balanced pipelining across the time consuming combinational blocks of the structure and projected an efficient fixed point implementation technique, also develop the steady-state error expression. The hardware design of LMS adaptive filter is proposed; to overcome this steady-state error researcher designed a noiseless tap LMS Adaptive filter.

Carlo Safarian et. al. [5] Discussed FPGA Implementation of LMS-based FIR Adaptive Filter for Real Time Digital Signal Processing Applications. Researcher tries to reduce the excess use of multipliers and longer cycle periods. Also proposed a design for an FPGA implementation of an LMS based adaptive filter using the Xilinx DSP48[5]. The proposed architecture uses one set of multipliers for both filter output and weight increment term calculation. The projected architecture consumes nearly 4.7 times less dynamic power than the available architecture. Therefore, the discussed architecture is suitable for efficient FPGA realization of an LMS FIR adaptive filter for real time DSP applications.

Abhishek Deb et. al. [9] gives and survey of Advanced Linear Adaptive Filtering Methods for Active Noise Control. In this survey, researcher discussed about the number of adaptive algorithms, which have different properties, but the 
main aim is to minimize the mean square error lessen the computational complexity and increase the convergence rate.

Boo-Shik Ryu et.al. [10] Discussed The Performance of an Adaptive Noise Canceller with DSP processor. In this paper, real time execution of the LMS based techniques on the DSK TMS320C6713 is presented. For a low order filter the NLMS obtained the maximum convergence speed along with best frequency and time performance. For a high filter order the results showed the conflicting. The VSS-LMS proved to have the maximum processing speed for the two cases, but the error graph showed that the NLMS had improved performance and in the FFT response showed various harmonic close to $2 \mathrm{KHz}$.

http://zone.ni.com/, [11] National instrument pvt. Ltd provides basic and useful information about adaptive filter and its algorithms.

Yen-Tai Lai, et. al. [12] in this technical article on 'Design and Implementation of an Adaptive FIR Filter Based on Delayed Error LMS Algorithm' shows how adaptive filtering scheme are generally used in the fields of signal processing and communication such as noise cancellation and speech coding. Adaptive filters usually need real time ability to process signal. The filter can be designed using the digital adaptive finite impulse response filter based on the delayed error least mean square algorithm. The architecture has good hardware utilization efficiency and it can be easily scaled the filter without reducing the throughput rate.

After brief analysis of Journals and articles, we have listed out some advantages and disadvantages mentioned in the table 1 . below.

Table -1. Literature comparison and there advantages and disadvantages

\begin{tabular}{|l|l|l|l|}
\hline Author & $\begin{array}{l}\text { Proposed } \\
\text { Technique }\end{array}$ & Advantage & Disadvantage \\
\hline $\begin{array}{l}\text { Pramod } \\
\text { kumar } \\
\text { meher }\end{array}$ & $\begin{array}{l}\text { efficient } \\
\text { architecture for } \\
\text { the } \\
\text { implementation } \\
\text { of DLMS } \\
\text { adaptive filter }\end{array}$ & $\begin{array}{l}\text { No fixed } \\
\text { point issues } \\
\text { and Less area } \\
\text { requirement. }\end{array}$ & $\begin{array}{l}\text { Fixed-point } \\
\text { implementatio } \\
\text { n issues and } \\
\text { Delay \& Area } \\
\text { high. }\end{array}$ \\
\hline $\begin{array}{l}\text { S. } \\
\text { Savitha } \\
\text { and S. } \\
\text { Lakshmi }\end{array}$ & $\begin{array}{l}\text { efficient LMS } \\
\text { adaptive filter } \\
\text { with low adaption } \\
\text { delay, for } \\
\text { achieving area } \\
\text { delay power and } \\
\text { lower adaption } \\
\text { delay }\end{array}$ & $\begin{array}{l}\text { Lower power } \\
\text { consumption } \\
\text { and compact } \\
\text { layout giving } \\
\text { smaller chip } \\
\text { area. }\end{array}$ & $\begin{array}{l}\text { fixed step size } \\
\text { parameter for } \\
\text { every } \\
\text { iteration }\end{array}$ \\
\hline
\end{tabular}

\begin{tabular}{|c|c|c|c|}
\hline $\begin{array}{l}\text { Carlo } \\
\text { Safarian }\end{array}$ & $\begin{array}{l}\text { FPGA } \\
\text { Implementation } \\
\text { of LMS-based } \\
\text { FIR Adaptive } \\
\text { Filter for Real } \\
\text { Time Digital } \\
\text { Signal Processing } \\
\text { Applications }\end{array}$ & $\begin{array}{l}\text { reduce the } \\
\text { excess use of } \\
\text { multipliers } \\
\text { and longer } \\
\text { cycle periods }\end{array}$ & $\begin{array}{l}\text { Slower speed, } \\
\text { high power } \\
\text { consumption. }\end{array}$ \\
\hline $\begin{array}{l}\text { Boo- } \\
\text { Shik } \\
\text { Ryu }\end{array}$ & $\begin{array}{l}\text { Performance of } \\
\text { an Adaptive } \\
\text { Noise Canceller } \\
\text { with DSP } \\
\text { processor }\end{array}$ & $\begin{array}{l}\text { obtained the } \\
\text { maximum } \\
\text { convergence } \\
\text { speed along } \\
\text { with best } \\
\text { frequency and } \\
\text { time } \\
\text { performance }\end{array}$ & $\begin{array}{l}\text { Speed of } \\
\text { convergence } \\
\text { and steady } \\
\text { state miss- } \\
\text { adjustment. }\end{array}$ \\
\hline
\end{tabular}

\section{COMPARISON BETWEEN ADAPTIVE} ALGORITHMS

With the help of section 2 background and motivations of research and literature survey, we compare the LMS and RLS algorithm and conclude which adaptive algorithm is suitable for adaptive filter. Both computational resource and convergence speed requirements when choosing an adaptive filter algorithm are important. The key difference is that LMS algorithm is a Markov process. It has its present state, but other than that it doesn't remember data from the past. For time varying signals this is a characteristic because past data will give you invalid information about the current parameters.

The RLS algorithm uses all of the information, present and past, but that can be a problem if the past data is ambiguous for the current parameters. If researcher looking for a quantitative rule for when to use one or the other, we don't have one. RLS algorithm is more computationally intensive than LMS algorithm, so if LMS is good enough, then that is the secure one to go with. RLS algorithm converges faster, but it is more computationally intensive and has the time varying disadvantage. In table 2. we compare the performance of adaptive algorithms and in table 3 . Computational resource requirements and relative convergence speed for different adaptive filter algorithms are given below.

Table 2. Performance Comparison of Adaptive Algorithm

\begin{tabular}{|c|c|c|c|}
\hline Algorithm & $\begin{array}{c}\text { Mean } \\
\text { square } \\
\text { error }\end{array}$ & complexity & stability \\
\hline LMS & $1.5 * 10-2$ & $2 \mathrm{~N}+1$ & Less Stable \\
\hline RLS & $6.2 * 10-3$ & $4 \mathrm{~N} 2$ & High Stable \\
\hline
\end{tabular}

Table 3. Computational resource requirements and relative convergence speed for different adaptive filter algorithms[11] Where, " $n$ " is the length of the adaptive filter, "M" is the length of the estimated impulse response of the resultant path.

\begin{tabular}{|c|c|c|c|c|c|c|}
\hline \multirow{2}{*}{ Algorithm } & \multicolumn{4}{|c|}{ Computational Resource } & \multirow{2}{*}{ Relative Convergence Speed } \\
\cline { 2 - 7 } & Usage of Memory & Multiplication & Addition & Division & Shift & \\
\hline LMS & $2 n$ & $2 n+1$ & $2 n+1$ & 0 & 0 & Fast \\
\hline
\end{tabular}




\begin{tabular}{|l|l|l|l|l|l|l|}
\hline Recursive Least Squares (RLS) & $n^{2}+2 n$ & $2\left(n^{2}+2 n\right)$ & $1.5 n^{2}+2.5 n$ & 0 & 0 & Very Fast \\
\hline
\end{tabular}

\section{CONCLUSION}

In this paper a survey is done on Adaptive filter and adaptive algorithms. Adaptive filter is a computational device that shows the relationship between the input and output signals of the filter. It self-adjusts the filter coefficients according to an adaptive algorithm. Many researcher and author works on the adaptive filter algorithms. After exhaustive literature survey shows in the above section all state of art methods and ideas where discussed. It is not enough to distinguish between different adaptive algorithms, hints paper has shown difference between various adaptive algorithms based on different parameters. This paper will guide new researchers to fetch clear information about adaptive filter and adaptive algorithms through a single paper. After comparison of many journals and articles we formulated that the relative coverage speed of LMS algorithms fast and RLS algorithms is very fast.

\section{REFERENCES}

[1] M. Sridhar and K. Sivanandam, "Low Adaptation Delay in Fixed Point LMS Adaptive Filter for DSP Applications," pp. 690-694, 2016.

[2] J. Dhiman, S. Ahmad, and K. Gulia, "Comparison between Adaptive filter Algorithms (LMS, NLMS and RLS)," Int. J. Sci. Eng. Technol. Res., vol. 2, no. 5, pp. 2278-7798, 2013.

[3] P. K. Meher and S. Y. Park, "Area-delay-power efficient fixed-point LMS adaptive filter with low adaptation-delay," IEEE Trans. Very Large Scale Integr. Syst., vol. 22, no. 2, pp. 362-371, 2014.

[4] D. N. Pearson and K. K. Parhi, "Low-power FIR digital filter architectures," Proc. - IEEE Int. Symp. Circuits Syst., vol. 1, pp. 23-26, 1995.
[5] C. Safarian, T. Ogunfunmi, and W. J. Kozacky, "FPGA Implementation of LMS-based FIR Adaptive Filter for Real Time Digital Signal Processing Applications,” pp. 12511255, 2015.

[6] R. P. Patil and P. P. Bharadkar, "Survey on Different Architectures of DLMS," pp. 838-842, 2015.

[7] A. C. Mugdha, F. S. Rawnaque, and M. U. Ahmed, "filter algorithm in noise removal from ECG signals," Informatics, Electron. Vis. (ICIEV), 2015 Int. Conf., pp. 16, 2015.

[8] M. S. Savitha and M. S. Lakshmi, "IMPLEMENTATION OF EFFICIENT LMS ADAPTIVE,” pp. 1414-1417, 2015.

[9] A. Deb, A. Kar, and M. Chandra, "Advanced linear adaptive filtering methods for active noise control: A technical survey," ICACCS 2015 - Proc. 2nd Int. Conf. Adv. Comput. Commun. Syst., pp. 3-7, 2015.

[10] B. Ryu, J. Lee, J. Kim, and C. Lee, "Performance of an adaptive noise canceller with DSP processor," IEEE, no. 1, pp. 42-45, 2008.

[11] National instrument, "Digital Filter Design Toolkit Help," 2014. [Online]. Available: http://zone.ni.com/reference/enXX/help/371988G-01/lvaftconcepts/aft_choose_algorithm/. [Accessed: 12-Dec-2016].

[12] Y. Kou, Y. Ai, and Z. Ma, "Design and Implementation of an Adaptive Interference Mitigation Algorithm Based on," pp. $360-371,2009$ 